\title{
Z-99 The quantitative capabilities of HEM inversion for the sea ice case
}

AUTHORS

A. PFAFFLING ${ }^{1}$ and J. E. REID ${ }^{2}$

Address

${ }^{1}$ Alfred Wegener Institute for Polar and Marine Research, Division Climate Physics, PO Box 12 0161, 27515

Bremerhaven, Germany

${ }^{2}$ University of Tasmania, School of Earth Sciences, Australia

\begin{abstract}
Summary
Semi-empirical methods are routinely used for Helicopter Electromagnetic (HEM) sea ice thickness mapping. Although these methods yield sufficiently accurate thickness data, it is of interest to determine whether formal one-dimensional (1D) geophysical inversion could yield improved results. If both the thickness and the ice conductivity could be mapped, the results could be used to estimate glaciological parameters such as the age of the sea ice. Sea ice conductivity data could also be potentially used to estimate the strength of the ice sheet, which would be valuable information for planning of icebreaking operations. By investigating synthetic and field data we show that, in the case of level sea ice of thickness up to $2 \mathrm{~m}$, the accuracy of our HEM system is not high enough to sense the small conductivity variations arising from the age of the ice. Sea ice conductivity has a stronger influence on the measured HEM responses in areas of thick, deformed sea ice (pressure ridges), where bulk conductivity is higher as a result of the large seawater-filled porosity. Synthetic three-dimensional HEM data generated for pressure ridge models has shown that the 1D interpretation methods conventionally used for interpretation of sea ice thickness overestimate the true bulk conductivity at $3 \mathrm{D}$ features.
\end{abstract}

\section{Introduction}

A semi empirical processing technique, termed 'EMPEX' [2], has been developed to estimate sea ice thickness from HEM data acquired using the Alfred Wegener Institute (AWI) dualfrequency system. The EMPEX method has been used operationally since 2001, and has been successfully applied to interpretation of HEM data from the Arctic, Antarctic and Baltic. The method relies on three main approximations:

1) The seawater conductivity is assumed to be known and constant.

This assumption is generally satisfied, as within a certain region the conductivity hardly varies within geophysical scales and it is always possible to obtain a water sample to measure the conductivity for that particular region. However, the variation between different geographical regions can be dramatic, being $\sim 2.7 \mathrm{~S} / \mathrm{m}$ for Arctic or Antarctic seawater, but only $0.3 \mathrm{~S} / \mathrm{m}$ for the brackish Baltic Sea.

2) The sea ice conductivity is assumed to be zero.

The contrast between water and sea ice is so pronounced $(2.7 \mathrm{~S} / \mathrm{m}$ to $<0.05 \mathrm{~S} / \mathrm{m})$ that varying the ice conductivity in 1D forward modeling hardly changes the modelled fields, especially for the lower frequency of the AWI system $(3.68 \mathrm{kHz})$. However, in the Arctic for example we meet young, saline first year ice (FY) and old, drained second- or multi year ice (MY) with bulk conductivities around $0.05 \mathrm{~S} / \mathrm{m}$ and $0.005 \mathrm{~S} / \mathrm{m}$ respectively. This is still a small difference compared to the contrast with seawater but the ability to distinguish these different ice types would be a significant improvement to the resulting dataset. 
3) The ice is considered to be one-dimensional.

As long as level (undeformed) sea ice is considered, this is true. In fact, the level ice thickness is the key parameter for climate change research and it has been shown, that EMPEX methods give reliable level ice thickness estimates, meeting the main claim for climate change studies. However, pressure ridges and floe edges are clearly 2D or 3D and influence the HEMdetermined ice thickness distribution.

Assessing these assumptions and evaluating the benefits of alternatives is presented in the following.

\section{Methods}

To evaluate the abilities of inversion for HEM sea ice thickness measurements, synthetic data were computed for 1D layered halfspace models as well as 3D models using MARCO_AIR developed by the Electromagnetic Modeling Group within CSIRO Exploration \& Mining [3]. Gaussian noise, with standard deviations estimated from field data, was added to the computed model responses.

A HEM field data set from East Antarctica, acquired during September-October 2003 is also considered. In this paper a $500 \mathrm{~m}$ profile is analyzed, as extensive ground truth data is available for this ice floe. The characteristics of this dataset are given in [2]. In addition to the nearly one thousand drilled thickness measurements done along the line, a series of Wenner resistivity soundings as well as DC anisotropy measurements were done and ice cores were taken to complete the geophysical ground truth picture.

For comparative reasons different processing algorithms were applied to both synthetic and field data:

1) EMPEX sea ice thickness determination.

The semi empirical EMPEX method is routinely used at AWI and was described and presented at Near Surface 2004 by the authors [2]. The EMPEX method uses a double exponential fit to a 1D halfspace model curve which is used to determine the bird height above the sea ice / seawater interface. Subtracting the laser altitude of the bird from this EM height directly yields the sea ice thickness. A detailed paper on the method is in preparation to be submitted to Near Surface Geophysics.

2) $1 \mathrm{D}$ least-squares inversion.

Least-squares 1-D inversion was conducted using the freeware Gauss-Marquardt-Levenberg nonlinear parameter estimation package PEST by Watermark Numerical Computing [1]. In the standard 1D inversion, HEM data were inverted assuming a two-layered model with the sea ice thickness and conductivity allowed to vary, and the seawater conductivity fixed. This is referred to as 'simultaneous 1D inversion' for the purposes of this paper.

3) SbS inversion.

Being a two frequency system, the AWI bird's measurements have different sensitivities to the sea ice thickness and ice conductivity. The low frequency $(3.68 \mathrm{kHz})$ is hardly affected at all by changes in the conductivity of the sea ice, and is controlled by the seawater conductivity. The high frequency $(112 \mathrm{kHz})$ data is however more sensitive to the sea ice conductivity. The sensitivities of the 3.68 and $112 \mathrm{kHz}$ in-phase (IP) to the ice conductivity are 80 and $136 \mathrm{ppm} /(\mathrm{S} / \mathrm{m})$ respectively for $2 \mathrm{~m}$ thick level ice.

Consequently a "step by step" ( $\mathrm{SbS}$ ) inversion approach was applied to the data. In the first step only the low frequency IP is considered to determine the ice thickness. For this stage of the inversion, the sea ice conductivity is fixed at zero. The second step in the algorithm is to fix the final, inverted thickness from the first step and invert the high frequency IP response for ice conductivity. Both steps are done using the PEST package as described above. The 
SbS inversion results in more stable conductivity estimates than the simultaneous $1 \mathrm{D}$ inversion, especially in areas of thicker ice.

\section{Results}

The results of the EMPEX processing of the field data from East Antarctica have been discussed in [2], and are plotted in Figure 1 as the black line. The known underestimation of the pressure ridge keel depth due to footprint [4] and 3D effects is obvious. Deviations from the drilled thickness (white line) along the level ice parts (especially between 100 and $220 \mathrm{~m}$ ) originate from bird movements like pitch and roll, which influence the induction geometry as well as the measured laser altitude.

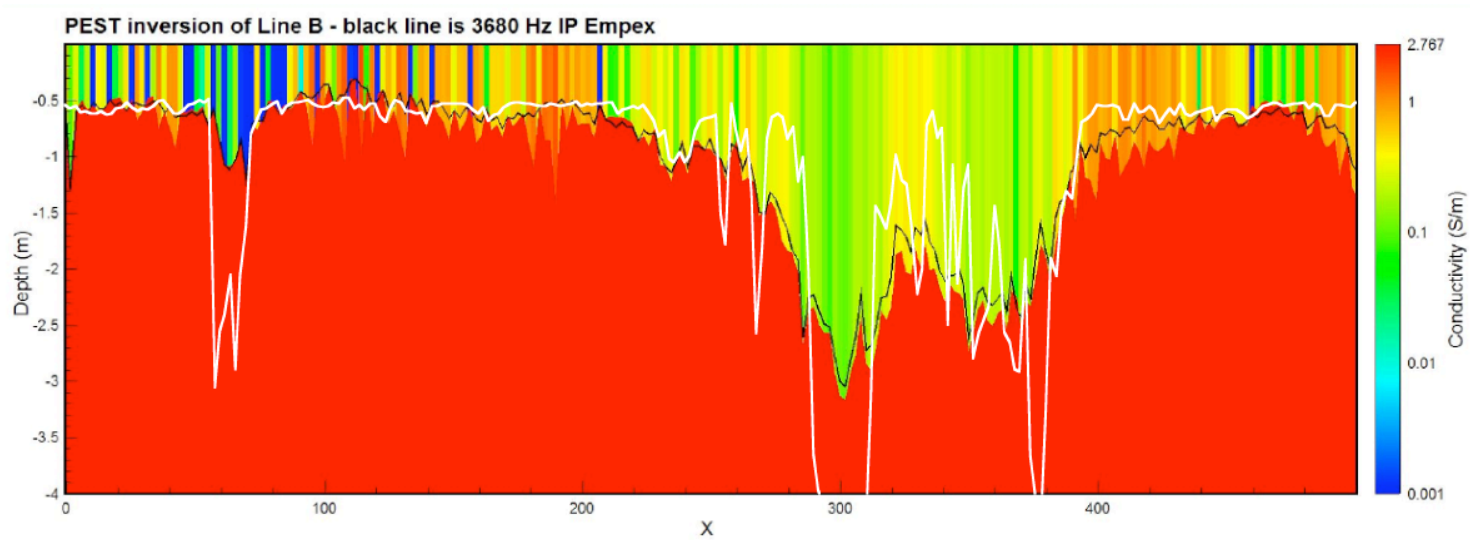

Figure 1: Simultaneous 1D inversion results compared with EMPEX (black line) and drilled thicknesses (white line). The starting model was a $2 \mathrm{~m}$ thick sea ice layer of conductivity $0.05 \mathrm{~S} / \mathrm{m}$, overlying seawater of conductivity $2.767 \mathrm{~S} / \mathrm{m}$.

The background color plot is the result of the simultaneous 1D inversion using the $3.68 \mathrm{kHz}$ IP \& Q and $112 \mathrm{kHz}$ IP data only. Seawater conductivity was known and was fixed at $2.767 \mathrm{~S} / \mathrm{m}$ for all inversions. The inverted thickness generally agrees well with the EMPEX results. Where the ice is thin, the inverted thickness shows more scatter than the EMPEX thickness which points out the sensitivity of the 1D inversion to both noise levels and to minor calibration errors in the data. The large variations in the recovered sea ice conductivity within the level ice between $0-200 \mathrm{~m}$ indicates that the HEM data are insensitive to the conductivity of such thin ice. This has been confirmed by inversion of noise-contaminated synthetic data for $0.5 \mathrm{~m}$ thick level ice with variable conductivity. Even for $2 \mathrm{~m}$ thick level ice, it is hard for the inversion to resolve conductivity effects given typical AWI HEM noise levels. It therefore appears that discrimination between FY and MY ice can not be achieved using HEM data.

A significant feature in the inversion result is the increased conductivity of the major pressure ridge keel between $280-400 \mathrm{~m}$. The high pressure ridge conductivity is reasonable from a glaciological standpoint, as FY Antarctic pressure ridges are composed of jumbled blocks of ice with extensive seawater-filled voids. From the geophysical point of view, however, it is possible that the increased conductivity within the pressure ridge is an artifact of $1 \mathrm{D}$ inversion of a $3 \mathrm{D}$ structure.

Figure 2 presents the results of the SbS inversion. Sea ice thickness and conductivity show less variability than in Figure 1, partly because the HEM data were smoothed using a 5-point moving average filter prior to the SbS inversion. However, even when data are not smoothed, both thickness and ice conductivity seem to be better modelled using the SbS method than with the simultaneous 1D approach. The pressure ridge keel appears as a zone of higher conductivity using both the SbS and simultaneous inversion methods. 


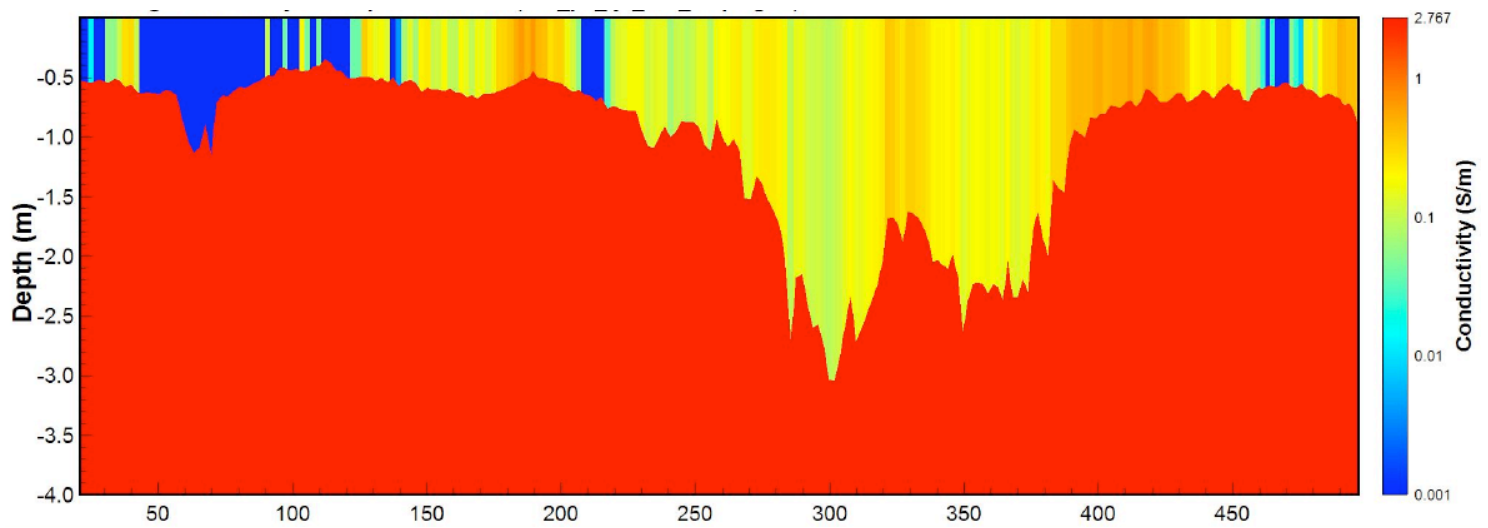

Figure 2: SbS inversion results of smoothed data. Starting model ice conductivity for the second step of the inversion was $2.77 \mathrm{~S} / \mathrm{m}$.

To answer the question of whether the high keel conductivity is reasonable or not, synthetic data from two 3D pressure ridge keel models, computed using MARCO_AIR, were interpreted using the simultaneous 1D approach. One 3D model had a keel conductivity of $0.05 \mathrm{~S} / \mathrm{m}$ (the same as the level ice surrounding the ridge) and the other had a keel conductivity of $0.2 \mathrm{~S} / \mathrm{m}$. In both cases, the conductivity recovered by the $1 \mathrm{D}$ inversion was higher than the true conductivity, indicating the presence of a 3D artifact in the 1D inversion results.

\section{Conclusions}

The simultaneous 1D and SbS inversion schemes show no significant advantage over the operational EMPEX method for deriving sea ice thickness from HEM data. Rather than improving the results, inversion introduces further challenges, being more sensitive to noise and imperfect system calibration.

Analysis of synthetic and field data shows, that it is not possible to detect small sea ice conductivity changes typical of first year and multi year ice via 1D inversion of HEM data. The expected higher conductivity of a pressure ridge keel cannot definitely be mapped, as the conductive effect is complicated by a 2D / 3D artifact.

\section{Acknowledgements}

Parts of this work were supported by the Antarctic Climate \& Ecosystems Cooperative Research Centre based in Hobart / Australia within the AWI - ACE CRC exchange programe, and by Australian Antarctic Science (AAS) grant \#2381.

\section{References}

[1] Doherty, J., 2003, Groundwater model calibration using Pilot Points and Regularisation: Ground Water, 41 (2), 170-177.

[2] Pfaffling, A., Haas, C. and Reid, J. E., 2004, Empirical inversion of HEM data for sea ice thickness mapping: Extended abstracts, 10th European Meeting of Environmental and Engineering Geophysics (EAGE's Near Surface 2004), Utrecht, The Netherlands.

[3] Raiche, A., 2001, Choosing an AEM system to look for kimberlites - a modelling study: Expl. Geophys., 32, 1-8.

[4] Reid, J. E., Pfaffling, A., Vrbancich, J., 2005, Airborne electromagnetic footprints in one-dimensional earths: submitted to Geophysics. 